\title{
A norbornyl route to azasugars: a new synthesis of deoxynojirimycin analogues
}

\author{
Goverdhan Mehta* and Narinder Mohal \\ Department of Organic Chemistry, Indian Institute of Science, Bangalore 560 012, India
}

\begin{abstract}
A new synthesis of deoxynojirimycin (DNJ) analogues (galacto- and altrose configuration) has been achieved through a functionalized cyclopentene derivative crafted from the norbornyl system, employing double reductive amination as the key step. The new DNJ analogues have been evaluated against various glycosidases and found to be moderate to strong inhibitors.
\end{abstract}

Keywords: azasugars; glycosidase inhibitors; osmylation; reductive amination.

Natural and unnatural polyhydroxylated piperidines have aroused the widespread attention of organic chemists in recent years due to their very promising biological activity profile and synthetically challenging structural features present in them. ${ }^{1}$ These polyhydroxylated piperidines generically termed as iminosugars ('azasugars'), closely resemble monosaccharides in terms of their shape and structure; they competitively inhibit glycosidases, enzymes responsible for the cleavage of glycosidic bonds. In azasugars, the ring oxygen is replaced by nitrogen, which can be protonated under physiological $\mathrm{pH}$, thus mimicking the glycopyranosyl cation. Inhibition of glycosidases is projected to be useful in the treatment of carbohydrate related metabolic disorders and holds promise for the development of drugs for the treatment of cancer, diabetes, HIV and viral infections. ${ }^{2}$ A large number of naturally occurring azasugars and their synthetically designed analogues are known, and nojirimycin $\mathbf{1}$, 1-deoxynojirimycin $\mathbf{2}^{\text {1a }}(\mathrm{DNJ})$, amidine $\mathbf{3}^{3 \mathrm{a}}$ and deoxyfuconojirimycin $\mathbf{4}^{3 \mathrm{~b}}$ are typical examples, all of which have been found to inhibit carbohydrate processing enzymes.
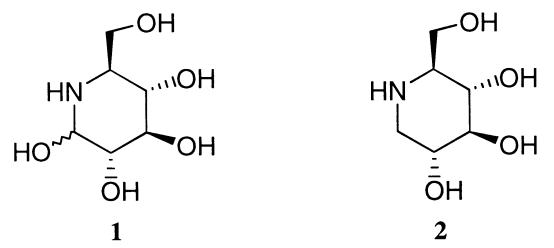<smiles>NC1=N[C@H](CO)[C@@H](O)[C@H](O)[C@H]1O</smiles><smiles>C[C@H]1NC[C@@H](O)[C@H](O)[C@@H]1O</smiles>

\footnotetext{
* Corresponding author. E-mail: gm@orgchem.iisc.ernet.in
} 
A wide range of synthetic strategies have been devised to access polyhydroxylated piperidines. ${ }^{1,3,4}$ Prominent among these, which are also of general applicability, are the restructuring or elaboration of chiral pool derived precursors (carbohydrates, amino acids, myo-inositol, tartaric acid etc.), ${ }^{1 \mathrm{a}, 3,4}$ chemoenzymatic ${ }^{4 \mathrm{~b}, \mathrm{c}}$ and microbial (hydroxylated aromatics) approaches, ${ }^{4 \mathrm{~d}}$ Diels-Alder ${ }^{4 e}$ and hetero-Diels-Alder ${ }^{4 f}$ cycloaddition based routes, and aza-Achmatowicz reaction (furan reorganization) ${ }^{4 \mathrm{~g}, \mathrm{~h}}$ Herein, we report a new synthesis of deoxynojirimycin analogues originating from a bicyclo[2.2.1] heptane (norbornyl) system.

Recently we have developed a fragmentation reaction-based strategy to extract a highly functionalized and stereochemically well defined cyclopentene derivative $\mathbf{8}$ from the readily available norbornyl derivative 5 through the intermediacy of 6 and 7, respectively, Scheme $1 .{ }^{5}$ Cyclopentane derivative 8 has now been further restructured to DNJ analogues ${ }^{6}$ employing double reductive amination as the key step.

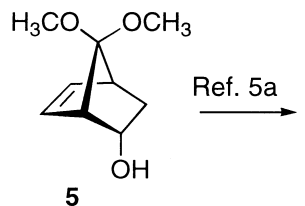

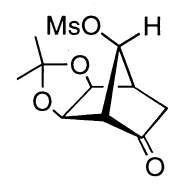

6

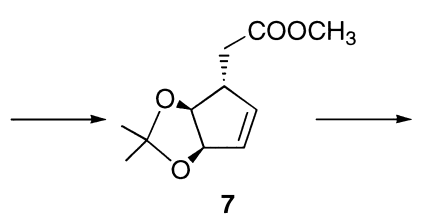

Scheme 1.

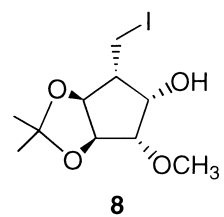

8

The free hydroxyl group in the iodocyclopentane $\mathbf{8}$ was transformed to a leaving group through mesylation to furnish the mesylate $\mathbf{9}$. Acetolysis of $\mathbf{9}$ led to elimination followed by allylic displacement to furnish acetoxycyclopentene 10, in which the olefinic moiety was well positioned for further manipulation. $\mathrm{OsO}_{4}$-mediated catalytic dihydroxylation of $\mathbf{1 0}$ proceeded in a stereoselective manner to give $\mathbf{1 1}^{7}$ and $\mathbf{1 2}^{\mathbf{7}}$ (10:90), Scheme 2. While diastereomers $\mathbf{1 1}$ and $\mathbf{1 2}$ were separated and characterized, it was not considered necessary for the next step. Sodium periodateinduced glycol cleavage in $\mathbf{1 1}$ and $\mathbf{1 2}$ furnished the labile keto-aldehyde $\mathbf{1 3}$ which was as such subjected to double-reductive amination in the $\mathrm{NaCNBH}_{3}-\mathrm{BnNH}_{2}$ milieu to furnish $\mathbf{1 4}$ as a

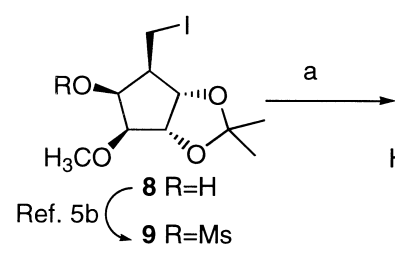

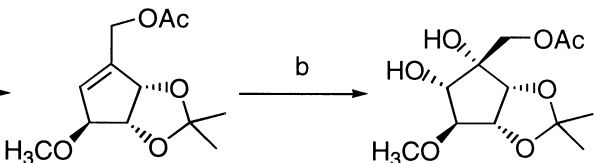

10
11

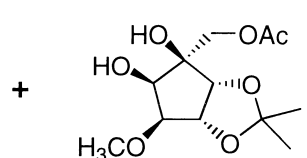

12<smiles>CO[C@H]1CN([C@@H](CO)c2ccccc2)[C@H]2OC(C)(C)O[C@@H]12</smiles>

16<smiles>CO[C@H]1C[NH2+][C@H](CO)[C@@H]2OC(C)(C)O[C@H]12</smiles>

15<smiles>CO[C@H]1CN(Cc2ccccc2)[C@H](COC(C)=O)[C@@H]2OC(C)(C)O[C@H]12</smiles>

14

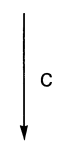

Scheme 2. Reagents and conditions: (a) $\mathrm{NaOAc}, \mathrm{DMF}, 105^{\circ} \mathrm{C}, 6 \mathrm{~h}, 77 \%$; (b) $\mathrm{OsO}_{4}, \mathrm{NMMO}\left(50 \%\right.$ aq. sol.), $\mathrm{Me}{ }_{2} \mathrm{CO}$ : $\mathrm{H}_{2} \mathrm{O}$ (4:1), 48 h, 86\%; (c) $\mathrm{NaIO}_{4}$ (1.3 equiv.), DCM, $0^{\circ} \mathrm{C}$; (d) $\mathrm{BnNH}_{2}, \mathrm{AcOH}, \mathrm{NaCNBH}_{3}, 20 \mathrm{~h},-10^{\circ} \mathrm{C} \rightarrow \mathrm{rt}, 30 \%$ for two steps; (e) $\mathrm{KOH}, \mathrm{MeOH}, 2$ h, $90 \%$ 
mixture of two diastereomers (1:2). Base mediated hydrolysis of the acetate group in $\mathbf{1 4}$ and chromatographic separation of the diastereomers led to $15^{7}$ and $16,{ }^{7}$ which were fully characterized as having the altrose and galactose stereochemical disposition, ${ }^{6}$ respectively, on the basis of incisive NMR (COSY, NOE) studies (Scheme 2).

In independent deprotection sequences, $\mathbf{1 5}$ and $\mathbf{1 6}$ were transformed to the hydrochlorides of $N$-benzyl DNJ derivatives $\mathbf{1 7}$ and 19, respectively, Scheme 3. In a similar manner, hydrochlorides of altro-deoxynojirimycin methyl ether $\mathbf{1 8}^{6}$ and galactostatin methyl ether $\mathbf{2 0}$ were obtained from 15 and 16 , respectively, and duly characterized. ${ }^{7}$

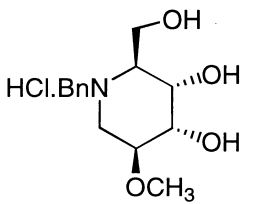

17<smiles>CO[C@H]1C[NH+]([SiH3])[C@H](CO)[C@H](O)[C@@H]1O</smiles>

19<smiles>CO[C@H]1CN[C@H](CO)[C@@H](O)[C@H]1O</smiles>

18<smiles>OC[C@H]1NC[C@@H](Cl)[C@@H](O)[C@@H]1O</smiles>

$20 \mathrm{R}=\mathrm{CH}_{3}$
$21 \mathrm{R}=\mathrm{H}$

Scheme 3. Reagents and conditions: (a) $2.5 \% \mathrm{HCl}: \mathrm{Et}_{2} \mathrm{O}(1: 1), 18 \mathrm{~h},>90 \%$; (b) $\mathrm{H}_{2}, \mathrm{Pd} / \mathrm{C}(10 \%), \mathrm{EtOH}, 18 \mathrm{~h}, 60 \%$; $2.5 \% \mathrm{HCl}: \mathrm{Et}_{2} \mathrm{O}(1: 1), 95 \%$; (c) same as (a), 90\%; (d) same as (b), quantitative

Table 1

Inhibition constants ${ }^{\mathrm{a}, \mathrm{b}}\left(\mathbf{K}_{\mathrm{i}}\right)$ in $\mu \mathrm{m}$

\begin{tabular}{|c|c|c|c|c|}
\hline \multirow[b]{2}{*}{ Enzyme } & \multicolumn{4}{|c|}{ compound } \\
\hline & 17 & 18 & 19 & 20 \\
\hline $\begin{array}{l}\alpha \text {-glucosidase } \\
\text { (yeast) }\end{array}$ & $*^{\mathrm{c}}$ & $\mathrm{NI}^{\mathrm{d}}$ & 784 & NI \\
\hline $\begin{array}{l}\beta \text {-glucosidase } \\
\text { (sweet almonds) }\end{array}$ & * & $\mathrm{NI}$ & NI & NI \\
\hline $\begin{array}{c}\alpha \text {-galactosidase } \\
\text { (green coffee beans) }\end{array}$ & * & 27 & 233 & 1.76 \\
\hline $\begin{array}{c}\beta \text {-galactosidase } \\
(\text { E. Coli })\end{array}$ & NI & $\mathrm{NI}$ & NI & NI \\
\hline
\end{tabular}

${ }^{\mathrm{a}}$ Each $200 \mu \mathrm{L}$ assay contained indicated enzyme $0.1-0.5 \mathrm{U} / \mathrm{mL}$, inhibitor $\mathbf{1 7 - 2 0}$ in water $(2-3 \mathrm{mM})$ and nitrophenyl glycosides $(2-2.5 \mathrm{mM})$ in appropriate buffer at optimal temp and $\mathrm{pH}$ of each enzyme. ${ }^{\mathrm{b}}$ Inhibition constants were determined using Dixon plots of inhibition data. ${ }^{c} 10-25 \%$ inhibition was observed at higher conc. (above 800 $\mu \mathrm{M}$ ) of inhibitor. ${ }^{\mathrm{d}}$ No inhibition is observed up to $1 \mathrm{mM}$ conc. of inhibitor. 
Compounds 17-20 were assayed for their glycosidase inhibition activity (Table 1). All measurements were carried out with the corresponding nitrophenyl glycoside substrates in aqueous buffer at appropriate $\mathrm{pH}$. Galactostatin methyl ether $\mathbf{2 0}$ was found to be a selective and potent inhibitor of $\alpha$-galactosidase and there was no inhibition observed for $\alpha$ - and $\beta$-glucosidases and $\beta$-galactosidase. It was notable that inhibitory activity of $\mathbf{2 0}$ was approximately 1000 fold less than of the natural product galactostatin 21. ${ }^{6}$ When $\mathbf{2 0}$ was compared with its benzyl derivative 19, a marked decline in inhibitory activity as well as selectivity was observed and the latter was only a moderate inhibitor of $\alpha$-galactosidase, and a weak inhibitor of $\alpha$-glucosidase. Interestingly, $18\left(\mathrm{C}_{2}\right.$-epimer of 20) was also found to be a selective inhibitor of $\alpha$-galactosidase whereas its benzyl derivative was found to be essentially ineffective towards glycosidase inhibition.

In short, we have devised a new synthesis of DNJ derivatives 17-20 from the cyclopentanoid building block 7. Our results of enzymatic assays reveal that $N$ - and $O$-substituents have significant consequence on the glycosidase activity and selectivity.

\section{Acknowledgements}

We thank Dr. Utpal Tatu of the Department of Biochemistry for useful discussions and valuable suggestions. N.M. thanks CSIR for the award of a Research Fellowship. We thank SIF, IISc, Bangalore for recording high field NMR.

\section{References}

1. Reviews: (a) Hughes, A. B.; Rudge, A. J. Nat. Prod. Reports 1994, 135 and references cited therein. (b) Ganem, B. Acc. Chem. Res. 1996, 29, 340. (c) Sears, P.; Wong, C.-H. Angew. Chem., Int. Ed. 1999, 38, 2300. (d) Heightman, T. D.; Vasella, A. T. Angew. Chem., Int. Ed. 1999, 38, 750.

2. Jacob, G. S. Curr. Opin. Struct. Biol. 1995, 5, 605 and references cited therein.

3. (a) Tong, M. K.; Papandreou, G.; Ganem, B. J. Am. Chem. Soc. 1990, 112, 6137. (b) Fleet, G. W.; Namguong, S. K.; Barker, C.; Bainses, S.; Jacob, G. S.; Winchester, B. Tetrahedron Lett. 1989, 30, 4439.

4. (a) Rudge, A. J.; Collins, I.; Holmes, A.; Baker, R. Angew. Chem., Int. Ed. Engl. 1994, 33, 2320 and references cited therein. (b) Straub, A.; Effenberger, F. J. Org. Chem. 1990, 55, 3926. (c) Kajimoto, T.; Liu, K. K.-C.; Pederson, L. R.; Zhong, Z.; Ichikawa, Y.; Porco Jr. J. A.; Wong, C.-H. J. Am. Chem. Soc. 1991, 113, 6187. (d) Johnson, C. R.; Johns, B. A. J. Org. Chem. 1997, 62, 6046. (e) Auberson, Y.; Vogel, P. Angew. Chem., Int. Ed. 1989, 28, 1498. (f) Streith, J.; Defoin, A. Synlett. 1996, 189. (g) Ciufolini, M. A.; Hermann, C. Y. W.; Dong, Q.; Shimzu, T.; Swaminathan, S.; Xi, N. ibid 1997, 105. (h) Martin, R.; Moyano, A.; Pericas, M. A.; Riera, A. Org. Lett. 2000, 2, 93.

5. (a) Mehta, G.; Mohal, N. Tetrahedron Lett. 1999, 40, 5791. (b) Mehta, G.; Mohal, N. Tetrahedron Lett. 1999, 40, 5795 .

6. For the isolation and enzyme inhibitory activity of the potent DNJ, deoxygalactostatin, see: Miyake, Y.; Ebata, M. J. J Antibiotics 1987, 40, 122. For the leading references towards the synthesis of deoxygalactostatin and analogues, see: (a) Paulson, H.; Hayauchi, Y.; Sinnwell, V. Chem. Ber. 1980, 113, 2601. (b) Bernotas, R.; Pezzone, M. A.; Ganem, B. Carbohydr. Res. 1987, 167, 305. (c) Furneaux, R. H.; Tyler, P. C.; Whitehouse, L. A. Tetrahedron Lett. 1993, 34, 3609. (d) Chida, N.; Tanikawa, T.; Tobe, T.; Ogawa, S. Chem. Commun. $1994,1247$. (e) Johnson, C. R.; Golebiowski, A.; Sundram, H.; Miller, M. W.; Dwaihy, R. L. Tetrahedron Lett. $1995,36,653$. (f) Barili, P. L.; Berti, G.; Catelani, G.; D’Andrea, F.; De Rensis, F.; Puccioni, L. Tetrahedron 1997, 53, 3407. (g) Shilvock, J. V.; Fleet, G. W. J. Synlett 1998, 554. (h) Asano, K.; Hakogi, T.; Iwana, S.; Katumura, S. Chem. Commun. 1999, 41. (i) Uriel, C.; Santoyo-Gonazaleaz, F. Synlett 1999, 593. (j) Ruiz, M.; Ruanova, T. M.; Ojea, V.; Quintela, J. M. Tetrahedron Lett. 1999, 40, 2021.

7. All new compounds reported here were racemic and gave satisfactory spectral data $\left({ }^{1} \mathrm{H}\right.$ and ${ }^{13} \mathrm{C} \mathrm{NMR}$, IR, Mass). Selected spectral data $\left({ }^{1} \mathrm{H}\right.$ NMR and $\left.{ }^{13} \mathrm{C} \mathrm{NMR}\right) 15 \delta_{\mathrm{H}}\left(300 \mathrm{MHz}, \mathrm{CDCl}_{3}\right): 7.32-7.28(5 \mathrm{H}, \mathrm{m}, \mathrm{Ar}-\mathrm{H}), 4.18(1 \mathrm{H}, \mathrm{dd}$, 
$J=2.7,6.0 \mathrm{~Hz}), 4.08(1 \mathrm{H}, \mathrm{dd}, J=4.8,6.0 \mathrm{~Hz}), 4.03\left(1 \mathrm{H}, \frac{1}{2} \mathrm{ABq}, J=13.2 \mathrm{~Hz}\right), 3.78\left(1 \mathrm{H}, \frac{1}{2} \mathrm{ABq}, J=13.2 \mathrm{~Hz}\right), 3.66$ $(1 \mathrm{H}, \mathrm{dd}, J=4.2,10 \mathrm{~Hz}), 3.54-3.45(3 \mathrm{H}$, series of m), $3.35(3 \mathrm{H}, \mathrm{s}), 3.15-3.10(1 \mathrm{H}, \mathrm{m}), 2.76(1 \mathrm{H}, \mathrm{dd}, J=3.3,13.5$ $\mathrm{Hz}), 2.62(1 \mathrm{H}, \mathrm{dd}, J=7.8,13.5 \mathrm{~Hz}), 1.56(3 \mathrm{H}, \mathrm{s}), 1.36(3 \mathrm{H}, \mathrm{s}) ; \delta_{\mathrm{C}}\left(75 \mathrm{MHz}, \mathrm{CDCl}_{3}\right): 138.72,128.88(2 \mathrm{C}), 128.51$ (2C), 127.42, 108.1, 76.43, 75.51, 75.01, 60.76, 60.46, 59.37, 57.20, 45.26, 28.06, 25.51. $16 \delta_{\mathrm{H}}\left(300 \mathrm{MHz}, \mathrm{CDCl}_{3}\right)$ : 7.34-7.26 (5H, m, Ar-H), $4.31(1 \mathrm{H}, \mathrm{dd}, J=2.2,6.0 \mathrm{~Hz}), 4.14(1 \mathrm{H}$, dd as t, $J=6.0 \mathrm{~Hz}), 4.12-4.10(2 \mathrm{H}, \mathrm{m}), 3.99-3.89$ $(2 \mathrm{H}$, series of $\mathrm{m}), 3.50-3.42(1 \mathrm{H}, \mathrm{m}), 3.42-3.38(1 \mathrm{H}, \mathrm{m}), 3.40(3 \mathrm{H}, \mathrm{s}), 3.0(1 \mathrm{H}, \mathrm{dd}, J=4.5,11.7 \mathrm{~Hz}), 2.68(1 \mathrm{H}, \mathrm{m})$, $1.91(1 \mathrm{H}, \mathrm{dd}, J=10.8,11.7 \mathrm{~Hz}), 1.57(3 \mathrm{H}, \mathrm{s}), 1.38(3 \mathrm{H}, \mathrm{s}) ; \delta_{\mathrm{C}}\left(75 \mathrm{MHz}, \mathrm{CDCl}_{3}\right): 137.73(\mathrm{C}), 129.14(\mathrm{CH}, 2 \mathrm{C})$, $128.35(\mathrm{CH}, 2 \mathrm{C}), 127.24(\mathrm{C}), 109.45(\mathrm{C}), 79.38(\mathrm{CH}), 78.96(\mathrm{CH}), 77.63(\mathrm{CH}), 61.71\left(\mathrm{CH}_{2}\right), 60.63(\mathrm{CH}), 57.86$ $\left(\mathrm{CH}_{3}\right), 56.69\left(\mathrm{CH}_{2}\right) 51.69\left(\mathrm{CH}_{2}\right), 28.28\left(\mathrm{CH}_{3}\right), 26.26\left(\mathrm{CH}_{3}\right) .17 \delta_{\mathrm{H}}\left(300 \mathrm{MHz}, \mathrm{D}_{2} \mathrm{O}\right): 7.41(5 \mathrm{H}$, br. s), 4.26-3.98 $(6 \mathrm{H}$, series of $\mathrm{m}), 3.55\left(1 \mathrm{H}\right.$, br. s), 3.4-3.08 $\left(3 \mathrm{H}\right.$, series of m), $3.16(3 \mathrm{H}, \mathrm{s}) ; \delta_{\mathrm{C}}\left(100 \mathrm{MHz}, \mathrm{D}_{2} \mathrm{O}\right): 132.84(2 \mathrm{C}), 131.55$, 130.57 (2C), 129.36, 76.23, 67.43, 65.96, 64.62, 63.68, 58.31, 55.81, 49.07. $18 \delta_{\mathrm{H}}\left(300 \mathrm{MHz}, \mathrm{D}_{2} \mathrm{O}\right): 4.0(1 \mathrm{H}, \mathrm{dd}$ as t, $J=3.6 \mathrm{~Hz}), 3.82(1 \mathrm{H}, \mathrm{dt}, J=3.6,12.3 \mathrm{~Hz}), 3.70-3.65(3 \mathrm{H}$, series of m), 3.38-3.34 $(1 \mathrm{H}, \mathrm{m}), 3.29(3 \mathrm{H}, \mathrm{s}), 3.25-3.12$ $\left(2 \mathrm{H}\right.$, series of m); $\delta_{\mathrm{C}}\left(100 \mathrm{MHz}, \mathrm{D}_{2} \mathrm{O}\right): 76.22(\mathrm{CH}), 67.40(\mathrm{CH}), 64.49(\mathrm{CH}), 59.00\left(\mathrm{CH}_{2}\right), 57.67\left(\mathrm{CH}_{3}\right), 56.37(\mathrm{CH})$, $41.36\left(\mathrm{CH}_{2}\right) .19 \delta_{\mathrm{H}}\left(300 \mathrm{MHz}, \mathrm{D}_{2} \mathrm{O}\right): 7.51(5 \mathrm{H}$, br. s), $4.26(2 \mathrm{H}, \mathrm{m}), 4.19-4.17(3 \mathrm{H}, \mathrm{m}), 3.68-3.60(2 \mathrm{H}, \mathrm{m}), 3.54$ $\left(1 \mathrm{H}\right.$, br. s), $3.43(1 \mathrm{H}, \mathrm{dd}, J=4.5,12.3 \mathrm{~Hz}), 3.30(3 \mathrm{H}, \mathrm{s}), 2.76(1 \mathrm{H}, \mathrm{dd}, J=10.5,12 \mathrm{~Hz}), \delta_{\mathrm{C}}\left(100 \mathrm{MHz}, \mathrm{D}_{2} \mathrm{O}\right): 132.85$ (2C), 131.64, 130.57 (2C), 129.45, 74.91, 72.75, 70.78, 65.92, 59.68, 59.44, 58.27, 50.63. 20: $\delta_{\mathrm{H}}\left(300 \mathrm{MHz}, \mathrm{D}_{2} \mathrm{O}\right)$ : 4.02-3.99 $(\mathrm{m}, 1 \mathrm{H}), 3.75-3.50(5 \mathrm{H}$, series of $\mathrm{m}), 3.34(3 \mathrm{H}, \mathrm{s}), 3.27-3.23(1 \mathrm{H}, \mathrm{m}), 2.70-2.62(1 \mathrm{H}, \mathrm{t} \mathrm{like} \mathrm{m}) ; \delta_{\mathrm{C}}(100$ $\left.\mathrm{MHz}, \mathrm{D}_{2} \mathrm{O}\right): 74.93(\mathrm{CH}), 72.56(\mathrm{CH}), 67.40(\mathrm{CH}), 60.53(\mathrm{CH}), 59.60\left(\mathrm{CH}_{2}\right), 58.85\left(\mathrm{CH}_{3}\right), 44.24\left(\mathrm{CH}_{2}\right)$. 